\title{
BCL-W expression associates with poor outcome in patients with peripheral T-cell lymphoma not otherwise specified
}

(c) The Author(s) 2021

Blood Cancer Journal (2021)11:153; https://doi.org/ 10.1038/s41408-021-00549-6

\section{To the Editor:}

The pro-survival BCL-2 family members $B C L-2, B C L-X L$ (BCL2L1), $B C L-W$ (BCL2L2), BCL-2A1 and $M C L-1$ contribute to tumor maintenance, progression, and chemo-resistance across a range of cancers, but their contributions in Peripheral T-cell Lymphoma Not Otherwise Specified (PTCL-NOS) are poorly understood [1]. $\mathrm{BH} 3$ profiling, is today considered the gold standard for prediction of $\mathrm{BCL}-2$ family proteins dependence. However, it can be performed only on fresh material and requires major expertise, available in only in limited centers $[2,3]$.

Direct evaluation of $\mathrm{BCL}-2$ alternative protein expression by immunohistochemistry (IHC) is a more commonly available approach, and can be performed on paraffin embedded samples [4]. In a series of 112 patients, each protein was detected by IHC in a subset of PTCLs, using a $10 \%$ cut-off, with variable expression based on PTCL subtypes (median, BCL-2 46\%, BCL-XL 57\%, and MCL-1 65\%) [5]. In a series of 173 patients with PTCL, BCL-2 expression was confirmed by IHC in $64 \%$ of cases, again with extreme variability across PTCL subtypes [6]. Therefore, the prognostic role of $\mathrm{BCL}-2$ expression in PTCL has been only partially explored, with conflicting reported data, and limited information regarding the prognostic role of other $\mathrm{BCL}-2$ family proteins $[7,8]$.

This is a retrospective analysis of patients with PTCL-NOS receiving either frontline or salvage therapy at the MD Anderson Cancer Center between 09/2000 and 09/2019, and for whom pretreatment tissue biopsy samples were available for correlative analysis. The 2014 Lugano Classification was retrospectively applied to determine treatment response in all patients [9]. The study was approved by the Institutional Review Board of The University of Texas MD Anderson Cancer Center and was conducted in accordance with the principles of the Declaration of Helsinki.

After routine diagnostic assessment, formalin-fixed paraffin embedded (FFPE) of lymph node and extranodal tissues were sectioned at a thickeness of $4 \mu \mathrm{m}$ and stained using Leica Bond RX automated stainer (Leica Biosystems). Primary antibody against BCL-2 (clone E17, cat\# ab32124, dilution 1:200), MCL-1 (clone D5V5L, cat\#39224S, dilution 1:100), BCL-W (polyclonal, cat\# AF8241, dilution 1:50), BCL-2A1 (polyclonal, cat\# ab45413, dilution $1: 200$ ), BCL-XL (clone 54H6, cat\# 2764, dilution 1:1500), Tbet (TBX21/Tbet, clone 4B10, cat\# ab91109, dilution 1:300), CXCR3 (clone 1C6/CXCR3, cat\# 557183, dilution 1:200), GATA3 (clone EPR166551, cat\# ab199428, concentration 1:250), and CCR4 (polyclonal, cat\# HPA031613, dilution 1:200) were used and incubated for $15 \mathrm{~min}$ at room temperature. The antibody was detected using Bond Polymer Refine Detection kit (Leica
Biosystems) with Diaminobenzidine (DAB) as chromogen. Tonsils were used as external positive controls and reactive immune cells were used as internal control. All cases were analyzed using standad microscopy by three expert hemato-pathologists. The BCL-2 family proteins scoring was assessed based on percentage of positive neoplastic cells (whole slide) and scored in 10\% increments. The cell-of-origin (COO) for PTCL-NOS (TBX21/Th1 versus GATA3/Th2 subgroup) was assessed using the algorithm proposed by Amador et al. [10].

Association with categorical variables was evaluated using $x 2$ or Fisher exact tests, as appropriate. The difference in a continuous variable between patient groups was evaluated by the MannWhitney test. Progression-free survival (PFS) was defined as time from start of therapy to progression of disease, death, or last followup (whichever occurred first), and censored at time fo stem cell transplant. OS was defined as time from start of therapy to death or last follow-up. PFS and OS were calculated for all patients in the study and for subgroups of patients using Kaplan-Meier estimates, and were compared between subgroups using the log rank test. Multivariable Cox regression analysis was performed to assess the associations between patient characteristics and PFS or OS. A $p$-value of $\leq 0.05$ (two-tailed) was considered statistically significant. Statistical analyses were completed using SPSS 21 and GraphPad Prism 8.

Twenty-seven patients with PTCL-NOS were included in the study, with a median age of 65 years (range, 22-87 years). Sixteen (59\%) patients were previously treated, with a median number of 2 systemic therapies (range, 1-7), including SCT in 5 (19\%; autologous in 4, allogeneic in 1 patient). Remaining baseline characteristics are shown in Table 1.

$\mathrm{COO}$ and $\mathrm{BCL}-2$ family proteins expression were assessed on all pre-treatment tissue samples by IHC. Fifteen (56\%) patients showed a TBX21/Th1 phenotype, 10 (37\%) GATA3/Th2 and 2 (7\%) were unclassifiable (Supplementary Fig. 1). The median expression of BCL-2, BCL-XL, BCL-W, BCL-2A1 and MCL-1 was $30 \%$ (range: $0-90 \%$ ), 10\% (range: 0-90\%), 100\% (range: $40-100 \%), 20 \%$ (range: $0-90 \%$ ), and $70 \%$ (range: $1-100 \%$ ), respectively (Supplementary Fig. 2). Of interest, only $B C L-2$ and $B C L-W$ were expressed in $100 \%$ of cells, in 11 and $67 \%$ of cases, respectively. BCL-2A1 expression was significantly higher in patients who were previously treated (median, $35 \%$ vs $5 \%, p=0.02$ ), and in those who had previously received 2 or more lines of therapy (median, $40 \%$ vs $5 \%, p=0.02)$; $\mathrm{BCL}-2$ expression was significantly higher in patients with a GATA3/Th2 phenotype (60\% vs $20 \%, p=0.05)$ (Fig. 1A-E). No significant difference in other BCL-2 family proteins expression was observed according to baseline characteristics.

Among the 27 patients included in this study, 24 (89\%) received treatment after tissue biopsy collection, including chemotherapy in $17(63 \%)$ and biological therapy in $7(16 \%)$, for a median of 2 cycles (range, 1-6); 3 (11\%) did not receive any further treatment. Overall, 19 (76\%) patients were evaluable for response, whereas 5 (24\%) died or discontinued subsequent therapy (due to toxicity) before first response assessment. CR was achieved in 5 (24\%) 
patients, and $6(22 \%)$ patients proceeded with subsequent consolidation with SCT, including autologous SCT in 4 patients and allogeneic in 2. A trend for higher BCL-W expression was

Table 1. Baseline characteristics of the patients with PTCL-NOS.

\begin{tabular}{|c|c|}
\hline Patients ( $\mathbf{N}=\mathbf{2 7}$ ) & Number (\%), median [range] \\
\hline Age $<65$ years & $13(48)$ \\
\hline$\geq 65$ years & $14(52)$ \\
\hline Female & $9(23)$ \\
\hline Male & $18(67)$ \\
\hline Stage I-III & $14(52)$ \\
\hline IV & $13(48)$ \\
\hline Previously untreated & $11(41)$ \\
\hline Previously treated & $16(59)$ \\
\hline Previous therapies $<2$ & $16(59)$ \\
\hline$\geq 2$ & $11(41)$ \\
\hline No previous SCT & $22(81)$ \\
\hline Previous SCT & $5(19)$ \\
\hline No previous XRT & $25(93)$ \\
\hline Previous XRT & $2(7)$ \\
\hline Th1 phenotype & $15(56)$ \\
\hline Th2 phenotype & $10(37)$ \\
\hline Unclassified & $2(7)$ \\
\hline MCL-1 (\%) & $70[1-100]$ \\
\hline BCL-W (\%) & $100[40-100]$ \\
\hline BCL-XL (\%) & $10[0-90]$ \\
\hline BCL-2A1 (\%) & $20[0-90]$ \\
\hline BCL-2 (\%) & $30[1-100]$ \\
\hline
\end{tabular}

SCT stem cell transplant, XRT radiotherapy, Th $\mathrm{T}$ helper. observed among patients not achieving CR (100\% vs $90 \%, p=$ 0.07). No significant difference in $\mathrm{BCL}-2$ family proteins expression was observed when comparing patients who achieved CR to those who did not.

After a median follow-up of 28 months (95\% confidence interval [Cl], 14-42 months), 22 (81\%) patients progressed and/or died, and median PFS was 4 months ( $95 \% \mathrm{Cl}, 2-6$ months). Factors associated with shorter median PFS on univariate analysis were high expression of BCL-W, defined as quartile 3-4 (3 vs 7 months, $p=0.001$ ) (Fig. 1F-I) and GATA3/Th2 phenotype (3 vs 6 months, $p=0.04$ ). No significant association between other baseline characteristics and PFS was observed. On multivariate analysis including both factors, the association with PFS was maintained only for high expression of BCL-W (hazard ratio [HR] $0.3,95 \% \mathrm{Cl} 0.08-0.9, p=0.04$ ).

At most recent follow-up, 17 (63\%) patients died, and median OS was 6 months (95\% Cl, 1-12 months). Factors associated with shorter OS on univariate analysis were high BCL-W expression (4 months vs not reached) (Fig. 1F-l), lack of consolidation with SCT (4 months vs not reached, $p=0.001$ ), and GATA3/Th2 phenotype (4 vs 15 months, $p=0.03$ ). No other significant association between other baseline characteristics and OS was observed.

On multivariate analysis, only lack of consolidation with SCT maintained a significant association with OS (HR $0.1,95 \% \mathrm{Cl}$ $0.01-0.9, p=0.04$ ).

In this study, a higher expression of $\mathrm{BCL}-2 \mathrm{~A} 1$ was observed in previously treated patients as compared to previously untreated patients. BCL-2A1 represents a common mechanism of acquired chemo-resistance, and its upregulation has been previously described in peripheral blood-derived CLL cell cultures treated with fludarabine $[11,12]$. Further functional studies aimed at assessing whether its inhibition may decrease chemorefractoriness in PTCL-NOS patients are warranted.

In our analysis, a higher $\mathrm{BCL}-2$ expression was observed among patients with a GATA3/Th2 phenotype. Serum levels of Th1 and Th2-type effector cells-related cytokines (IL2, IFNY, IL4, IL10, IL5, IL6, IL1 $\beta$, TNFa, IL8) and soluble receptors (sIL2R, sIL6R) and BCL-2 proteins levels have been previously measured in a group of

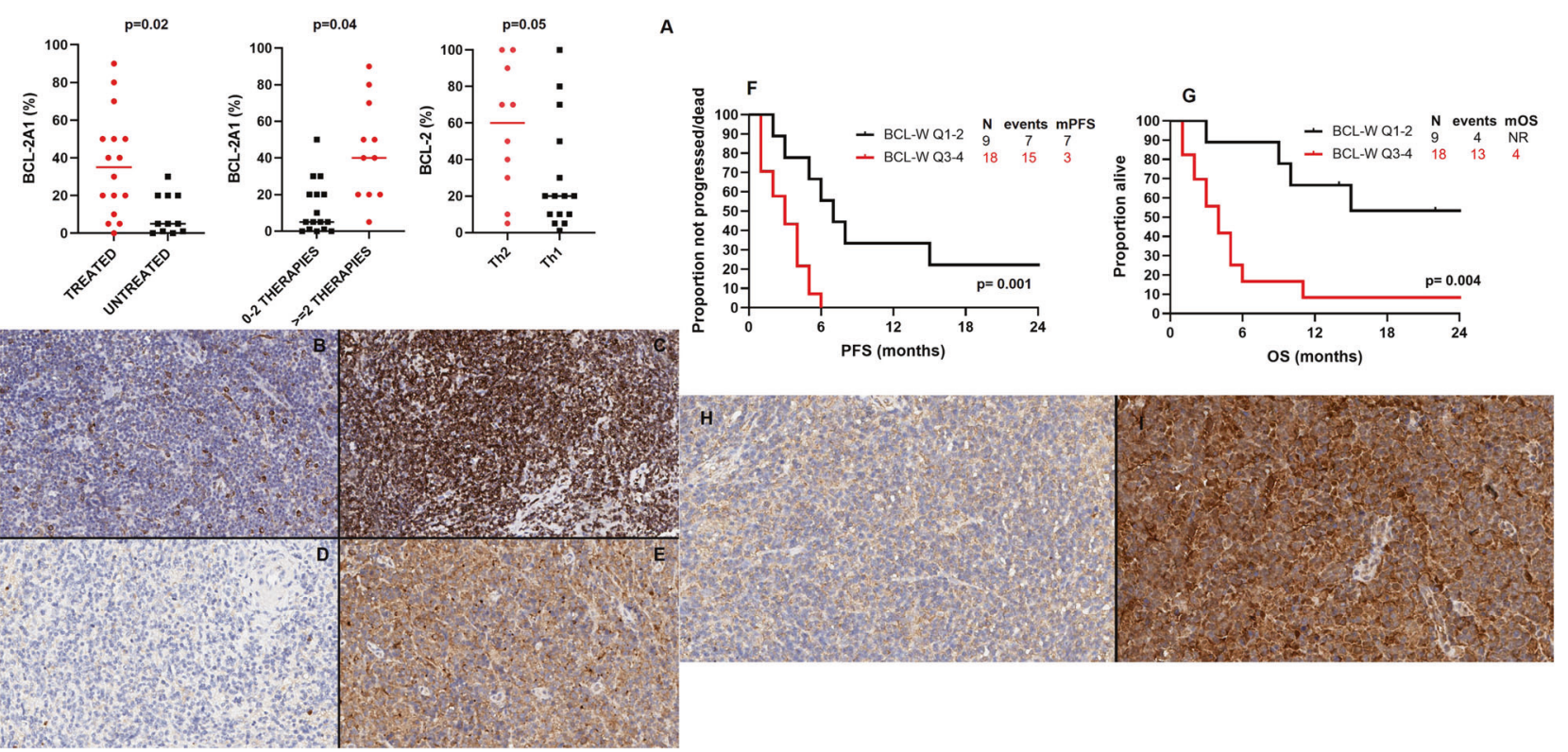

Fig. 1 Association between BCL-2 family proteins expression and baseline characteristics, and survival according to BCL-W expression. A Association between BCL-2A1 levels and previous therapy, and BCL-2 lvels and Th2 phenotype. B BCL-2 immunostain shows strong positivity in only $10 \%$ of lymphoma cells (low expression). C BCL-2 immunostain shows strong expression in 100\% of lymphoma cells (high expression). D BCL-2A1 immunostain shows negativity in all lymphoma cells (low expression). E BCL-2A1 immunostain shows diffuse and moderate to strong cytoplasmic expression in $80 \%$ of lymphoma cells (high expression). $\mathbf{F}$ Progression-free survival according to BCL-W expression. G Overall survival according to BCL-W expression. H BCL-W immunostain shows weak cytoplasmic positivity in $40 \%$ of lymphoma cells (low expression). I BCL-W immunostain shows diffuse and intense expression in $100 \%$ of lymphoma cells (high expression). 
healthy subjects, showing an association between GATA3/Th2 phenotype and higher levels of BCL-2 [13]. These findings suggest that further investigation of the efficacy of BCL-2 inhibitors, such as venetoclax, in patients with PTCL-NOS and GATA3/Th2 phenotype may help identify better treatment strategies for this subgroup with otherwise very poor outcome.

Of interest, independent of GAT3/Th2 phenotype, increased BCL-W expression was associated with shorter PFS in our study. Functional studies aimed at confirming the negative prognostic impact played by BCL-W expression in PTCL-NOS observed in this study are warranted, while functional dependence on $B C L-W$ for PTCL-NOS has yet to be proven [14].

In our analysis, the negative prognostic impact of $B C L-W$ expression on OS was trumped by the use of SCT as a subsequent consolidation strategy. However, PTCL-NOS patients are frequently SCT-ineligible or experience sub-optimal outcomes with SCT, due to inhability to achieve $C R$ before conditioning: novel agents, able to increase $C R$ rate in these patients, are therefore desperately needed. Low doses of navitoclax have been investigated in combination with venetoclax in patients with relapsed or refractory T-cell acute lymphoblastic leukemia/lymphoma, showing better tolerability and an impressive $56 \%$ CR rate [15]. The development of more specific and safer BCL-W inhibitors and their investigation in PTCL pre-clinical models is needed.

We acknowledge the multiple limitations of this study, including its single center and retrospective nature, and its relatively small sample size, and lack of $\mathrm{BH} 3$ profiling data.

While clinical trials investigating the safety and efficacy of BCL-2 inhibition in PTCL-NOS are ongoing, these results suggest that concomitant BCL-W inhibition may be beneficial, and functional studies aimed at confirming these findings are highly needed.

\section{Mario L. Marques-Piubelli (D) ${ }^{1,5}$, Luisa M. Solis ${ }^{1,5}$, Edwin R. Parra ${ }^{1}$,} Luis Malpica Castillo ${ }^{2}$, Sushanth Gouni ${ }^{2}$, Ranjit Nair ${ }^{2}$, Dai Chihara (D) ${ }^{2}$, Marina Konopleva (iD ${ }^{3}$, Ignacio I. Wistuba ${ }^{\prime}$ Swaminathan P. lyer ${ }^{2}$, Francisco Vega (iD ${ }^{4,6}$ and Paolo Strati (iD) ${ }^{1,2,6}$ ${ }^{1}$ Department of Translational Molecular Pathology, The University of Texas MD Anderson Cancer Center, Houston, TX, USA. ${ }^{2}$ Department of Lymphoma and Myeloma, The University of Texas MD Anderson Cancer Center, Houston, TX, USA. ${ }^{3}$ Department of Leukemia, The University of Texas MD Anderson Cancer Center, Houston, TX, USA.

${ }^{4}$ Department of Hematopathology, The University of Texas MD Anderson Cancer Center, Houston, TX, USA. ${ }^{5}$ These authors contributed equally: Mario L. Marques-Piubelli, Luisa M Solis. ${ }^{6}$ These authors jointly supervised this work: Francisco Vega and Paolo Strati.

$$
\text { 凶email: pstrati@mdanderson.org }
$$

\section{DATA AVAILABILITY}

The data that support the findings of this study are available from the corresponding author upon reasonable request.

\section{REFERENCES}

1. Youle RJ, Strasser A. The BCL-2 protein family: opposing activities that mediate cell death. Nat Rev Mol Cell Biol. 2008;9:47-59.

2. Del Gaizo Moore V, Letai A. BH3 profiling-measuring integrated function of the mitochondrial apoptotic pathway to predict cell fate decisions. Cancer Lett. 2013;332:202-5.

3. Pollyea DA, Amaya M, Strati P, Konopleva MY. Venetoclax for AML: changing the treatment paradigm. Blood Adv. 2019;3:4326-35.

4. Marques-Piubelli ML, Schlette EJ, Khoury JD, Furqan F, Vega F, Soto LMS, et al. Expression of $\mathrm{BCL} 2$ alternative proteins and association with outcome in CLL patients treated with venetoclax. Leukemia Lymphoma. 2020;62:1129-35.

5. Rassidakis GZ, Jones D, Lai R, Ramalingam P, Sarris AH, McDonnell TJ, et al. BCL-2 family proteins in peripheral T-cell lymphomas: correlation with tumour apoptosis and proliferation. J Pathol. 2003;200:240-8.
6. Zaja F, Tabanelli V, Agostinelli C, Calleri A, Chiappella A, Varettoni M, et al. CD38, BCL-2, PD-1, and PD-1L expression in nodal peripheral T-cell lymphoma: Possible biomarkers for novel targeted therapies? Am J Hematol. 2017;92:E1-E2.

7. Li HL, Huang XP, Zhou XH, Ji TH, Wu ZQ, Wang ZQ, et al. Correlation of seven biological factors (Hsp90a, p53, MDM2, Bcl-2, Bax, Cytochrome C, and Cleaved caspase3) with clinical outcomes of ALK + anaplastic large-cell lymphoma. Biomed Environ Sci. 2011;24:630-41.

8. Julamanee J, Kayasut K, Lekhakula A. The expressions of P53, BCl-2, and P-glycoprotein and prognostic impact in patients with peripheral T-cell lymphoma (PTCL). J Med Assoc Thai. 2015;98:950-6.

9. Cheson BD, Fisher Rl, Barrington SF, Cavalli F, Schwartz LH, Zucca E, et al. Recommendations for initial evaluation, staging, and response assessment of Hodgkin and non-Hodgkin lymphoma: the Lugano classification. J Clin Oncol. 2014;32:3059-68.

10. Amador C, Greiner TC, Heavican TB, Smith LM, Galvis KT, Lone W, et al. Reproducing the molecular subclassification of peripheral T-cell lymphoma-NOS by immunohistochemistry. Blood 2019;134:2159-70.

11. Vogler $M$, Butterworth $M$, Majid $A$, Walewska RJ, Sun XM, Dyer MJ, et al. Concurrent up-regulation of $B C L-X L$ and $B C L 2 A 1$ induces approximately 1000-fold resistance to ABT-737 in chronic lymphocytic leukemia. Blood 2009;113:4403-13.

12. Olsson A, Norberg M, Okvist A, Derkow K, Choudhury A, Tobin G, et al. Upregulation of bfl-1 is a potential mechanism of chemoresistance in B-cell chronic lymphocytic leukaemia. Br J cancer. 2007;97:769-77.

13. Contasta I, Pellegrini P, Berghella AM, Adorno D. Cell cycle control in cellular homeostasis during the immune response: interactions between $\mathrm{TH} 1, \mathrm{TH} 2$ cytokines, and $\mathrm{Bcl} 2$ and p53 molecules. Cancer biotherapy radiopharmaceuticals. 2001;16:63-71.

14. Koch R, Brem E, Clark R, Kupper TS, Letai A, Weinstock DM. A functional characterization of $\mathrm{BCL} 2$-family members identifies $\mathrm{BH} 3$ mimetics as potential therapeutics in T-cell lymphomas. Blood 2016;128:22-292.

15. Pullarkat VA, Lacayo NJ, Jabbour E, Rubnitz JE, Bajel A, Laetsch TW, et al. Venetoclax and navitoclax in combination with chemotherapy in patients with relapsed or refractory acute lymphoblastic leukemia and lymphoblastic lymphoma. Cancer Disco. 2021;11:1440-53.

\section{ACKNOWLEDGEMENTS}

The authors thank the technical support of the following laboratory members: Mei Jiang, Wei Lu, Leticia Hamana, Khaja Kan, and Jianling Zhou. The study was partially supported by MD Anderson Cancer Center Support grant CA016672. PS salary is supported by the Lymphoma Research Foundation Career Development Award.

\section{AUTHOR CONTRIBUTIONS}

PS designed the study, analyzed the data and wrote the manuscript; SG analyzed the data and coauthored the manuscript; LMC, RN, DC, MK and SPI provided clinical care to patients and coauthored the manuscript; MLM-P, LMS, ERPC, IIW and FV performed IHC staining and analysis and coauthored the manuscript.

\section{COMPETING INTERESTS}

PS is a consultant for Genentech-Roche and has received research support from AstraZeneca. FV received research funding/support from National Cancer Institute, CRISP Therapeutics, Geron Therapeutics, and honoraria from 13 Health, Elsevier, America Registry of Pathology, Congressionally Directed Medical Research Program, and Society of Hematology Oncology. The remaining authors declare no competing interests.

\section{ETHICS APPROVAL}

This study was approved by the MD Anderson Cancer Center Institutional Review Board.

\section{ADDITIONAL INFORMATION}

Supplementary information The online version contains supplementary material available at https://doi.org/10.1038/s41408-021-00549-6.

Correspondence and requests for materials should be addressed to Paolo Strati.

Reprints and permission information is available at http://www.nature.com/ reprints

Publisher's note Springer Nature remains neutral with regard to jurisdictional claims in published maps and institutional affiliations. 
Open Access This article is licensed under a Creative Commons (cc) Attribution 4.0 International License, which permits use, sharing, appropriate credit to the original author(s) and the source, provide a link to the Creative Commons license, and indicate if changes were made. The images or other third party material in this article are included in the article's Creative Commons license, unless indicated otherwise in a credit line to the material. If material is not included in the article's Creative Commons license and your intended use is not permitted by statutory regulation or exceeds the permitted use, you will need to obtain permission directly from the copyright holder. To view a copy of this license, visit http://creativecommons. org/licenses/by/4.0/.

(c) The Author(s) 2021 